RESEARCH

\title{
THE IMPACT OF CLOSURE DEVICES ON VASCULAR COMPLICATIONS DURING TRANSCATHETER AORTIC VALVE IMPLANTATION PROCEDURES IN GERIATRIC PATIENTS
}

Turkish Journal of Geriatrics
DOI: $10.31086 /$ tjgeri.2021.216

2021; 24(2): 204-211

- Latif ÜSTÜNEL ${ }^{1}$

- Ibrahim Murat ÖZGÜLER ${ }^{1}$

CORRESPONDANCE

${ }^{1}$ Latif ÜSTÜNEL

Firat University Medical School, Cardiovascular Surgery, Elazig, Turkey

Phone: +905324548937

e-mail: latifustunel@hotmail.com

Received: Nov 27, 2020

Accepted: Mar 30, 2021

${ }^{1}$ Firat University Medical School Cardiovascular Surgery, Elazig, Turkey

\section{Abstract}

Introduction: In transcatheter aortic valve implantation, some closure devices can be used as an alternative to the surgical exploration of the femoral artery for vascular access. This study aimed to compare closure devices and surgical exploration techniques in terms of vascular access complications, particularly in geriatric patients.

Materials and Methods: This study included 169 patients who had undergone transcatheter aortic valve implantation at a cardiology clinic between August 2014 and January 2019. The patients' mean age was 76.62 years; $53.30 \%$ were male. The preferred access site was the femoral artery in all the patients. Patients in whom a closure device was used were included in the Prostar and ProGlide groups, and those who underwent surgical exploration of the femoral artery were included in the Surgery group. The endpoints of the study were postoperative bleeding, infection, and a recurring need for surgical repair.

Results: Of the patients, $55.60 \%, 29.60 \%$, and $14.80 \%$ were included in the Prostar, ProGlide, and Surgery groups, respectively. No significant differences were observed between the Prostar and ProGlide groups regarding the rate of need for emergency surgery due to bleeding. During post-procedure followups, the emergency surgery and bleeding rates were significantly higher in the Prostar and ProGlide groups than those in the Surgery group.

Conclusion: Although no difference exists between the closure devices used for vascular access in terms of complications, surgical exploration reduces the rate of bleeding-related complications thereby reducing the complication risk, except for the possible occurrence of surgical site infections.

Keywords: Transcatheter Aortic Valve Replacement; Vascular Closure Device; Femoral Artery 


\section{INTRODUCTION}

Transcatheter aortic valve replacement (TAVR) is an alternative to conventional aortic valve replacement in patients with symptomatic severe aortic stenosis (1). Transcatheter aortic valve implantation (TAVI) was first adopted in 2002. Since then, it has been increasingly used globally to reduce mortality and morbidity in patients with high or prohibitive surgical risks (2). In the recent years, TAVI has also been frequently and successfully performed as an alternative to the cardiopulmonary bypass (CPB) technique, which is currently used for aortic valve replacement, particularly in geriatric patients. The elderly population is a rapidly growing demographic characteristic of Western countries. By 2050, the global number of individuals aged $\geq 60$ years is expected to reach nearly 2 billion, and the population aged $\geq 80$ years is projected to exhibit a 26 -fold increase (3).

According to the Guidelines on Valvular Heart Diseases published in 2017 by the European Society of Cardiology (ESC), TAVR should be considered a primary treatment option in patients with symptomatic severe aortic stenosis who also have a history of porcelain aorta, radiation therapy, and sternotomy along with a Society of Thoracic Surgeons or Euro SCORE II $>4 \%$ and a logistics Euro SCORE I $>10 \%$ (4).

During the initial days of the employment of the TAVR technique, various methods have been used for vascular access, including the transapical approach (5). The transfemoral approach is recommended by international guidelines owing to its advantages in terms of reduced procedure time and risk of cerebrovascular injuries. $(4,6)$. Although various closure devices are used in TAVR, Prostar and ProGlide (Abbott Vascular Inc., Santa Clara, CA, US) are the most commonly used devices. Surgical exploration is another option in cases of anatomical unsuitability, device failure, or vascular complications.

This study aimed to compare the two different closure devices used for access in TAVI procedures that are commonly performed worldwide, particularly in geriatric patients using the open approach, that is, femoral artery exploration, in terms of mortality and morbidity. Impairment of the vascular structure, especially in elderly patients, can reduce the success of these closure devices, thereby leading to bleeding or the need for emergency surgery. In elderly patients, repairing the artery used as an access route for TAVI with closure devices provides benefits such as shorter operative time, less sedation, and lower surgical stress. Moreover, reduction of risk factors is vital in this age group, as geriatric patients are often considerably frail. In patients with unsuitable vascular structures for closure devices (anterior artery calcification and plaque structure), accessing the artery with surgical exploration and performing primary repair after the procedure appears to minimize potential complications.

\section{MATERIALS AND METHODS}

Patients who were symptomatic owing to severe aortic valve stenosis with comorbidities and in whom conventional surgery posed a high risk were evaluated for TAVR by the cardiology and cardiovascular surgery clinics in a single center. Anatomical features were evaluated using computed tomography (CT) and coronary angiography. The patients were informed if they were suitable for TAVR, and the procedure was planned by the heart surgery team. Ethical approval was obtained from the local ethics committee (27.09.2019-350399), and patient recruitment was initiated.

The data of 189 patients who underwent TAVR between August 2014 and January 2019 were retrospectively analyzed. The first 10 patients in the closure device group were excluded to minimize the statistical effects of the learning curve. A total of 169 patients were included in the study. The mean age was 76.62 years (standard deviation [SD]: 6.30) with an age range of 65-92 years, and 90 of the patients were male (53.30\%). All patients were considered 
to be in the geriatric age group. The patients were divided into three groups according to vascular closure type: Prostar, ProGlide, and Surgery. For TAVR, three types of transcatheter valves (Edwards Sapien, Edwards Lifescienses Corporation, Irvine, CA, USA), Abbott Symetis (Abbott Laboratories, Illinois, USA), and Medtronic CoreValve (Medtronic Corp. Minneapolis, MN, USA) were used. The endpoints for vascular access were the need for emergent re-exploration for bleeding and groin infection. All patients were contacted for follow-up checks on the $15^{\text {th }}$ day and at the first and third months after the intervention. The patients were examined for access site complications, heart rhythm, and echocardiography was performed.

\section{Statistical Analysis}

The Statistical Package for the Social Sciences (SPSS 21, Chicago, IL, USA) was used for the data analysis. The distribution of continuous variables was determined using the Kolmogorov-Smirnov, and Shapiro-Wilk normality tests in the data analysis. Parametric data were expressed as mean \pm standard deviation values, whereas nonparametric data were expressed as median (minimum-maximum) and categorical data were expressed as percentage values. The Kruskal-Wallis test was used to compare more than two groups that did not conform to the normal distribution. The Mann-Whitney $U$ test was used to determine the relationships between the two groups. The chi-square test (crosstab) was used to compare categorical data, and statistical significance was set at $p<0.05$. The characteristics of the groups and comorbidities are presented in Table 1.

\section{RESULTS}

For TAVR, the Edwards Sapien, Abbott Symetis, and Medtronic CoreValve transcatheter valves were used in 116 (68.60\%), 32(18.90\%), and 21 (12.40\%) patients, respectively. The Prostar group consisted of 94 (55.60\%) patients, and the ProGlide group consisted of 50 (29.60\%) patients. The remaining 25 (14.80\%) patients underwent surgical exploration of the femoral artery and primary closure because of unfavorable vascular anatomy. The mean age of this group was 76.62 years. The mean EuroSCORE was 7.40 , and the valve types and rates in the groups are provided in Table 2.

There was no significant difference in the rates of need for emergency surgery due to bleeding between groups 1 and 2 ( $p=0.292$ ). The rates of postoperative emergency surgery and bleeding were significantly higher in both groups that were treated with closure devices than in group 3 ( $p=$ 0.001) (Table 2).

There was a history of peripheral arterial disease in 66 patients, of whom 26 (56.50\%) underwent emergency surgery because of bleeding. This value was statistically significant ( $p=0.007)$ (Figure 1).

There was a history of diabetes mellitus in $96 \mathrm{pa}-$ tients, of whom 32 (33.33\%) underwent emergency surgery because of bleeding. This value was statistically significant $(p=0.009)$ (Figure 1$)$.

There was a history of transient ischemic attacks in 36 patients, of whom 23 (63.90\%) underwent emergency surgery because of bleeding. This value was also statistically significant $(P=0.006)$ (Figure 1$)$.

Figure 1. Comparison of bleeding events between the etiological factors of the three groups

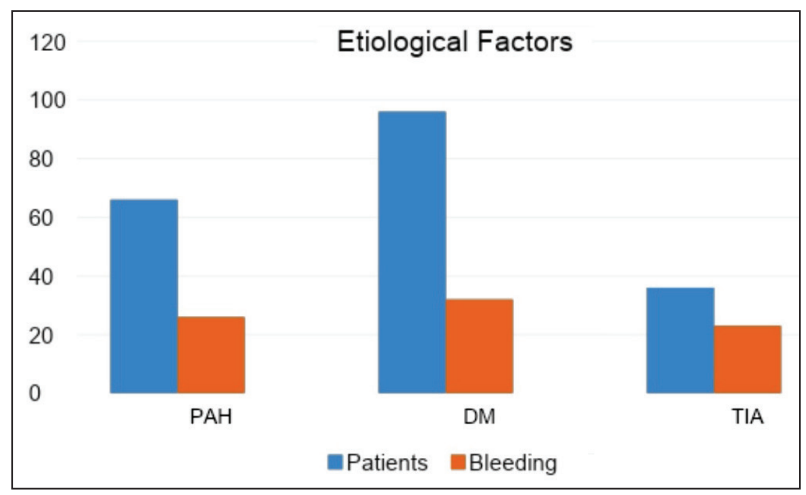


Table 1. Preoperative demographics and surgical characteristics of the patients

\begin{tabular}{|c|c|c|c|c|}
\hline & Group 1 & Group 2 & Group 3 & $\mathbf{P}$ \\
\hline Sex (M/F) & $94(47 / 47)$ & $50(27 / 23)$ & $25(16 / 9)$ & 0.456 \\
\hline Age (years) & 76.06 & 77.30 & 77.36 & 0.412 \\
\hline Ejection Fraction & 38.19 & 38.30 & 34.60 & 0.105 \\
\hline Body Mass Index & 27.15 & 27.04 & 27.06 & 0.123 \\
\hline EuroSCORE & 7.40 & 7.12 & 7.22 & 0.101 \\
\hline $\begin{array}{l}\text { Chronic Obstructive Pulmonary } \\
\text { Disease } \mathrm{n}(\%)\end{array}$ & $41(43.60)$ & $20(40)$ & $13(52)$ & 0.613 \\
\hline Reoperation & $7(7.40)$ & $4(8)$ & $3(12)$ & 0.761 \\
\hline Hypertension & $51(54.30)$ & $28(56)$ & $13(52)$ & 0.946 \\
\hline Aortic Regurgitation & $2(2.1)$ & $2(4)$ & $4(16)$ & 0.014 \\
\hline Coronary Disease & $28(29.80)$ & $16(32)$ & $9(36)$ & 0.832 \\
\hline Chronic Renal Failure & $10(10.60)$ & $4(8)$ & $3(12)$ & 0.830 \\
\hline Peripheral Arterial Disease & $40(42.60)$ & $14(28)$ & $12(48)$ & 0.143 \\
\hline Diabetes Mellitus & $47(50)$ & $33(66)$ & $16(64)$ & 0.134 \\
\hline Transiant Ischemic Attack & 30 (31.90) & $6(12)$ & 0 & 0.001 \\
\hline
\end{tabular}

\section{DISCUSSION}

Vascular complications, which are frequently observed during TAVR, may affect early mortality and morbidity. Suture-based closure devices generally shorten the operative time and reduce bleeding-related vascular complications (7-9). The effectiveness of such devices varies depending on the diameter of the access artery, device quality, presence of a calcified plaque structure, and diameter of the system to be used. There are many problems related to vascular structures that can negatively impact the effectiveness of these closure devices, especially in geriatric patients (10). Therefore, exploration of the femoral artery, which is an alternative option, combined with primary repair of the entry site, is the safest option, and features the lowest complication rate in the patients in this study. Few studies have compared the results obtained using Prostar and ProGlide. To the best of our knowledge, no study has compared the results of the two devices with the results of artery exploration and primary repair. In addition to comparing both devices, this study also compared the effects of arterial exploration and primary repair, a procedure that is considered more reliable in terms of preventing complications, on the mortality and morbidity of patients using these devices. This study showed that the complication rates of both closure devices were similar. However, the rates of bleeding and emergency surgical complications, excluding postoperative entry site infection, were significantly higher than those in the exploration and primary repair groups. 
Table 2. Valve types, times, and postoperative details in all groups

\begin{tabular}{|l|c|c|c|c|}
\hline & Group 1 & Group 2 & Group 3 \\
\hline Sapien & $76.6 \%(n=72)$ & $76 \%(n=38)$ & $24 \%(n=6)$ & P \\
\hline Symetis & $9.6 \%(n=9)$ & $12 \%(n=6)$ & $88 \%(n=17)$ & $0 \%(n=2)$ \\
\hline CoreValve & $13.80 \%(n=13)$ & $12 \%(n=6)$ & $0 \wedge$ & $0.292^{\star}, 0001^{\wedge}$ \\
\hline Bleeding & $32(34)^{\star}$ & $14(28)^{\star}$ & $82^{\wedge}$ & $0.610^{\star}, 0.001^{\wedge}$ \\
\hline Operative Time $(\min )$ & $54^{\star}$ & $57^{\star}$ & $2(8)$ & 0.001 \\
\hline Infection & 0 & 0 & $2(8)$ & \\
\hline Mortality & $7(7.4)$ & $3(6)$ & \\
\hline
\end{tabular}

Major and minor complications of TAVI-related vascular interventions are listed in the VARC II classification. These include stenosis, occlusion, dissection, surgical repair, suture rupture, bleeding, the need for endovascular stenting, and pseudoaneurysm formation $(11,12)$. The rates of these complications were $34 \%(n=32)$ in the Prostar group and $28 \%(n=14)$ in the ProGlide group. There were no complications in the surgical exploration group. The complication rates reported for both devices were comparable to those reported in previous studies $(13,14)$. However, it is noteworthy that no complications were observed in the surgery group, in which only two patients had surgical site infections during the postoperative period. Nevertheless, their length of hospital stay was higher than that of the other two groups, with 10 days of regular antibiotic use and wound care.

The mean age and EuroSCORE of the patient population were similar to those of patients who underwent the TAVI procedure in similar clinics and fell within the range required to qualify for official health insurance coverage in Turkey (15). The mean follow-up period for the patients was 12 months, which is believed to be sufficient for comparing mortality and morbidity beyond the length of hospital stay. There were no significant correlations between the EuroSCORE values and the rates of bleeding and emergency surgery $(p=0.101)$.

A previous study showed that the sex of the patient influenced the rate of TAVI complications; women were at greater risk (16). This increased risk is thought to stem from the smaller vessel diameter and the fact that vessels can rupture more easily in women (17). According to the results of the present study, there was no significant difference between men and women with respect to complications ( $p=$ 0.456 , chi-square test).

Many studies have reported that anticoagulation, peripheral artery disease, or the EuroSCORE value do not have a significant effect on vascular complications on their own $(2,18,19)$. In this study, no significant difference was detected in intergroup comparisons of patients with peripheral artery disease, but the rate of complications such as bleeding and the need for emergency surgery was found to be significantly higher according to the intragroup comparison. It is recommended that patients who are on long-term anticoagulants should be treated with TAVI through open exploration instead of using a closure device. Owing to the atherosclerotic and fragile vascular structure in geriatric patients, being selective in terms of preferred access to the 
femoral artery for TAVI is essential. CT angiography images should be evaluated in detail, and the use of closure devices through the femoral artery access route should be the first option if no calcification is detected in the anterior wall of the femoral artery. When diffuse calcification and plaque are detected on CT examination, closure devices should not be insisted on, and surgical exploration of the femoral artery followed by primary repair of the artery entry site should be performed with local anesthesia and sedation. This would prolong the operative time by nearly 20 minutes but would lead to a significant increase in mortality and morbidity.

This study also showed that although there was no significant difference between the patients with peripheral artery disease included in each of the closure device groups according to the intergroup comparison, the rates of bleeding and the need for emergency surgery were significantly higher than those found in intragroup comparisons. In our opinion, the sole presence of peripheral artery disease does not require an open exploration approach. However, the presence of a calcified plaque at the access site extending to the anterior wall or lateral walls according to the tomographic examination requires the use of a closure device, which is effective in relation to the calcification of the posterior wall of the access vessel. Therefore, all patients should be examined thoroughly to evaluate vascular structures before the procedure $(20,21)$.

Another result obtained in our study was that in patients with diabetes mellitus, the rates of bleeding and the need for emergency surgery were significantly higher in the groups treated with closure devices. Since damage to the vascular structure caused by diabetes is well known, TAVI with open exploration and primary repair for diabetic patients is also recommended $(22,23)$.

Bleeding is known to have a significant effect on 12-month mortality; it is the most important complication of closure devices $(24,25)$. Although many studies have shown that Prostar causes life-threat- ening bleeding more frequently than ProGlide, in the present study, there was no significant difference between the two groups.

A closure device was used in 144 patients, and 46 patients underwent urgent medical intervention. Of these 46 patients, 32 were in the Prostar group and 14 in the ProGlide group. Furthermore, 25 patients who were previously treated with femoral exploration did not require emergency medical intervention after primary repair. Recovery problems related to the wound site were identified in only two of the 25 patients; accordingly, the length of hospital stay was extended to $8-12$ days. There was no significant difference between these patients in terms of the 12-month postoperative mortality.

No clear data have been found to determine what lies beneath a vascular complication in the literature, but possibilities can be foreseen to a far enough extent to allow a cardiac team of cardiologists and cardiovascular surgeons, when combined with their clinical experience, to make a TAVI decision. In all patients, access angles should be observed with CT angiography for calcifications, plaques, artery wall irregularities, and folds at the access site, particularly in geriatric patients. Surgical exploration of the femoral artery and subsequent primary repair is believed to be more effective because the diameter of the system is wider in some valve models, especially in patients who will receive valve no. 29. Furthermore, we believe that closure materials should not be used in cases where the diameter of the femoral artery is less than $8 \mathrm{~mm}$, particularly when the anterior or lateral walls are covered by a calcified plaque. In our opinion, posterior wall calcification alone does not prevent the use of closure materials, and the effectiveness of closure devices is decreased in patients with a high skin-artery distance, high body mass index, and thick subcutaneous adipose tissue.

This study had some limitations, including its retrospective and non-randomized design. The sample size was relatively smaller than that in previous 
reports. Nevertheless, the researchers attempted to avoid bias in patient selection. Second, the distribution of the devices used for vascular closure was not homogenous because the Prostar device was more frequently used, particularly in early-stage TAVI implantations at our center, whereas the use of the ProGlide device was started later. Therefore, our surgeons had more experience with the Prostar device. Third, the sheath diameter was only altered in the Edwards Sapien valves, although it is known that different sheath sizes could lead to varying extents of vascular injury. Fourth, there was no significant difference between the groups with respect to the baseline characteristics. However, there was a trend toward male patients, which could influence the course of vascular complications and mortality.

Although vascular closure devices provide shorter operative times and allow local anesthesia, patients may require surgical re-exploration for vascular complications. There were no differences between the two devices in terms of complications. We suggest that the type of intervention should be

\section{REFERENCES}

1. Leon MB, Smith CR, Mack M, et al. Transcatheter aortic-valve implantation for aortic stenosis in patients who cannot undergo surgery. N Engl J Med 2010;363 (17): 1597-607. (PMID: 20961243)

2. Hayashida K, Lefèvre T, Chevalier B, et al. Transfemoral aortic valve implantation: New criteria to predict vascular complications. JACC Cardiovasc Interv 2011;4 (8): 851-8. (PMID: 21851897)

3. Yaffee DW, Williams MR. Cardiovascular Surgery in the Elderly. Sem in Thorac and Cardiovasc Surg 2016;28 (4): 741-47. (PMID: 28417859)

4. Baumgartner H, Falk V, Bax JJ, et al. 2017 ESC/ EACTS Guidelines for the management of valvular heart disease. Eur Heart J 2017;38 (36): 2739-91. (PMID: 28886619)

5. Walther T, Falk V, Borger MA, et al. Minimally invasive transapical beating heart aortic valve implantation - proof of concept. Eur J Cardio Thorac Surg 2007;31 (1): 9-15. (PMID: 17097302) selected according to the risks posed by anesthesia and groin complications.

\section{ACKNOWLEDGMENTS}

We would like to thank the Chief of Cardiology Department Prof. Ilgın Karaca, the Chief of Cardiovascular Surgery, Prof. Oktay Burma, and Associate Professor Ayhan Uysal for their interest. We would also like to thank the employees of Editage Corporation who played a role in the design and translation of this study.

\section{FUNDING}

This research was not funded by any public funding agency or by any commercial or nonprofit organizations.

\section{DECLARATION OF INTEREST}

None of the academic staff members at the Cardiology Department of our hospital have any conflicts of interest to declare.

6. Leon MB, Smith CR, Mack MJ, et al. Transcatheter or surgical aortic-valve replacement in intermediate-risk patients. N Engl J Med 2016;374 (17): 160920. (PMID: 27040324)

7. Overtchouk P, Modine T. Alternate access for TAVI: Stay clear of the chest. Interv Cardiol 2018;13 (3):14550. (PMID: 30443273)

8. Nishimura RA, Otto CM, Bonow RO, et al. AHA/ACC focused update of the 2014 AHA/ACC guideline for the management of patients with valvular heart disease: a report of the American College of Cardiology/American Heart Association Task Force on Clinical Practice Guidelines. JACC 2017;70 (2):25289. (PMID: 28315732)

9. Tchetche D, de Biase C, Brochado B, et al. How to make the TAVI pathway more efficient. Interv Cardiol 2019;14 (1):31-3. (PMID: 30858889)

10. Xianglai $X$, Brian W, Changhong R, et al. Age-related Impairment of Vascular Structure and Functions. Aging Dis 2017;8 (5):590-610. (PMID: 28966804) 
11. Kappetein AP, Head SJ, Généreux P, et al. Updated standardized endpoint definitions for transcatheter aortic valve implantation: The Valve Academic Research Consortium-2 consensus document (VARC2). JACC 2012;60 (15):1438-54. (PMID: 23036636)

12. Stehli J, Ji Q, Koh S, Duffy J, et al. Comparison of Outcomes of Transcatheter Aortic Valve Implantation in Patients Aged $>90$ Years Versus <90 Years. JACC 2019; 124 (7):1085-90. (PMID: 31353006)

13. Griese DP, Reents W, Diegeler A, Kerber S, BabinEbell J. Simple, effective and safe vascular access site closure with the double-ProGlide preclose technique in 162 patients receiving transfemoral transcatheter aortic valve implantation. Catheter Cardiovasc Interv 2013;82 (5): 734-41. (PMID: 23765732)

14. Hayashida K, Lefèvre T, Chevalier B, et al. True percutaneous approach for transfemoral aortic valve implantation using the Prostar XL device: Impact of learning curve on vascular problems. JACC Cardiovasc Interv 2012;5 (2):207-14. (PMID: 22361606)

15. Kappetein AP, Head SJ, Généreux $P$, et al. Updated standardized endpoint definitions for transcatheter aortic valve implantation: The Valve Academic Research Consortium-2 consensus document. JACC 2012;60 (15):1438-54. (PMID: 23036636)

16. Stangl V, Baldenhofer G, Laule M, Baumann G, Stangl K. Influence of sex on outcome following transcatheter aortic valve implantation (TAVI): Systematic review and meta-analysis. J Interv Cardiol 2014;27 (6):531-9. (PMID: 25156031)

17. Buchanan GL, Chieffo A, Montorfano M, et al. The role of sex on VARC outcomes following transcatheter aortic valve implantation with both Edwards SAPIEN and Medtronic CoreValve ReValving System ${ }^{\circledR}$ devices: The Milan registry. Euro Intervention 2011;7 (5):556-63. (PMID: 21930459)
18. Borz B, Durand E, Tron C, et al. Expandable sheath for transfemoral transcatheter aortic valve replacement: procedural outcomes and complication. Catheter Cardiovasc Interv 2014;83 (6):227-32. (PMID: 24403004)

19. Alnasser SM, Akshay B, Sanjit SJ, et al. Transradial approach for coronary angiography and intervention in the elderly: Metaanalysis of 777,841 patients. Int Journ of Card 2017; 228:45-51. (PMID: 27863361)

20. Brevetti G, Schiano V, Chiariello M. Endothelial dysfunction: A key to the pathophysiology and natural history of peripheral arterial disease? Atherosclerosis 2008;197 (1):1-11. (PMID: 18076886)

21. Alique M, Torres MPR, Bodega G, Noci MV, Troyano $\mathrm{N}$, et al. Microvesicles from the plasma of elderly subjects and from senescent endothelial cells promote vascular calcification. Aging (Albany NY). 2017; 9(3): 778-89. (PMID: 28278131)

22. Tousoulis D, Kampoli AM, Christodoulos S. Diabetes mellitus and vascular endothelial dysfunction: current perspectives. Curr Vasc Pharm 2012;10 (1):19-32. (PMID: 22112354)

23. Kalyani RR, Golden SH, Cefalu WT. Diabetes and Aging: Unique Considerations and Goals of Care. Diabetes Care 2017; 40(4): 440-3. (PMID: 28325794)

24. Amabile N, Azmoun A, Ghostine S, et al. Incidence, predictors and prognostic value of serious hemorrhagic complications following transcatheter aortic valve implantation. Int J Cardiol 2013;168 (1):151-6. (PMID: 23079085)

25. Pilgrim T, Stortecky S, Luterbacher F, Windecker S, Wenaweser P. Transcatheter aortic valve implantation and bleeding: incidence, predictors and prognosis. J Thromb Thrombolysis 2013;35 (4):456-62. (PMID: 23143652) 